\title{
Educational Program for Radiation Emergency Medicine at the Hirosaki University Graduate School of Health Sciences: A Training Course for Medical Personnel
}

\author{
Yoko Saito*, Toshiya Nakamura, Mayumi Urushizaka, Yu Kitajima, Chieko Itaki, Shingo Terashima, Yoichiro Hosokawa \\ Hirosaki University Graduate School of Health Sciences, Hirosaki, Japan
}

\section{Note}

Received July 17, 2015

Revision September 11, 2016

Accepted September 29, 2016

Corresponding author: Yoko Saito

Hirosaki University Graduate School of Health Sciences, 66-1 Hon-cho, Hirosaki, Aomori 036-8564, Japan

Tel: +81-172-39-5939

Fax: +81-172-39-5939

E-mail: yokosait@hirosaki-u.ac.jp

This is an Open-Access article distributed under the terms of the Creative Commons Attribution NonCommercial License (http://creativecommons.org/ licenses/by-nc/4.0) which permits unrestricted noncommercial use, distribution, and reproduction in any medium, provided the original work is properly cited.

Copyright $\odot$ 2016 The Korean Association for Radiation Protection

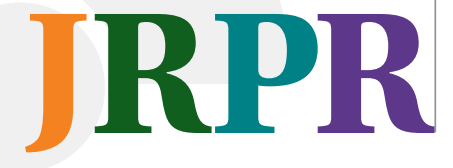

Background: Although nuclear disaster is considered rare, its effects are serious, and we must prepare a system to enable an effective response.

Materials and Methods: Since 2010, we have been offering a two-day seminar to provide current nurses and radiological technologists with basic knowledge and train them in radiation emergency medicine (REM) techniques. This training offers lectures to deepen each specialty from the perspective of REM, as well as exercises on ways to handle irradiated and/or contaminated patients. Participants were expected to treat patients according to the concept of REM.

Results and Discussion: All participants learn to assess and decontaminate contaminated wounds through drills. The questionnaire survey for participants indicated that participants were satisfied with this training and wanted to attend again.

Conclusion: We believe that this training course will provide a valuable opportunity for medical professionals to gain knowledge and expertise in REM.

Keywords: Radiation emergency medicine, Training course, Nurses, Radiological technologists

\section{Introduction}

Because several nuclear-related institutions are located in Aomori Prefecture, the prefectural government has designated Hirosaki University Hospital as the local tertiary radiation emergency hospital. In 2008, our university initiated the Radiation Emergency Medicine Project ${ }^{1)}$ and in 2010, established the Institute of Radiation Emergency Medicine $^{2)}$ and Emergency and Disaster Medical Center with radiation emergency medicine facilities ${ }^{3)}$ in Hirosaki University Hospital. Moreover, our faculty (i.e., Hirosaki University Graduate School of Health Sciences) has been developing human resources

\footnotetext{
1) Advanced Human Resources Development Project for Radiation Emergency Medicine. Background: History and Aim of the Project. (internet homepage. Hirosaki University Graduate School of Health Sciences, Special Expenditures Project of Ministry of Education, Culture, Sports, Science and Technology, Advanced Human Resources Development Project for Radiation Emergency Medicine)(cited September 06, 2016). Available from:http://www. hs.hirosaki-u.ac.jp/ hibaku/en/history.html\#001.

2) Japanese reaction to Fukushima Daiichi nuclear disaster, Wikipedia, 2015.

3) Emergency and Disaster Medical Center. (internet homepage. University Hospital, Institution and Facilities, Hirosaki University) (cited September 06, 2016) Available from: http://www.hirosaki-u.ac.jp/en/institution/hospital. html.
} 
for radiation emergency medicine since 2008, with the support of the Ministry of Education, Culture, Sports, Science and Technology of Japan. Subsequently, we introduced Radiation Emergency Medicine (REM)-related subjects to undergraduate and graduate education. Since 2010, our faculty has also been offering a short-term education program to provide current nurses and radiological technologists with basic knowledge and train them in REM techniques. ${ }^{4)}$ We organize a committee to manage this training program. In this paper, we provide an outline of this education program for working professionals.

\section{Materials and Methods}

\section{Concept of training program}

This training offers lectures to deepen each specialty from the perspective of REM, as well as exercises on ways to handle irradiated and/or contaminated patients. This training was originally offered as a three-day program; however, since 2013, we have incorporated prior learning through e-learning, and have shortened the contact course to a two-day program. The programs offered comprise a Nursing program and a Radiological Technologist program. The number of participants recruited was approximately 20 across both programs. A certificate of completion is issued to all program participants.

\section{Educational objectives}

The educational objective of this program is to foster nurses' and radiological technologists' knowledge and management ability necessary for REM and enable them to achieve appropriate correspondence and cooperation with each

Table 1. Training Course Program

\begin{tabular}{llll}
\hline E-learning & & 2-day course & \\
\hline Lecture & Radiation Basics* & Mental Health Problems in NPP Accident & A history of Serious Radiation Incidents \\
& Basic Radiation Biology* & Current Situation in Fukushima Prefecture \\
& Basic Radiation Protection* & Radiation Detection (Hands-on) & Decontamination (Drill) \\
& The Concept of REM & Hands- on/Drills & Team Organization (Desk Top Drill) \\
Movies & How To Put on/Take off Protective Clothing & NPP Accident Medical Treatment Drill & Medical Treatment Drill Review \\
& Radiation Patients Treatment &
\end{tabular}

*Not required in Radiological Technologists Course.
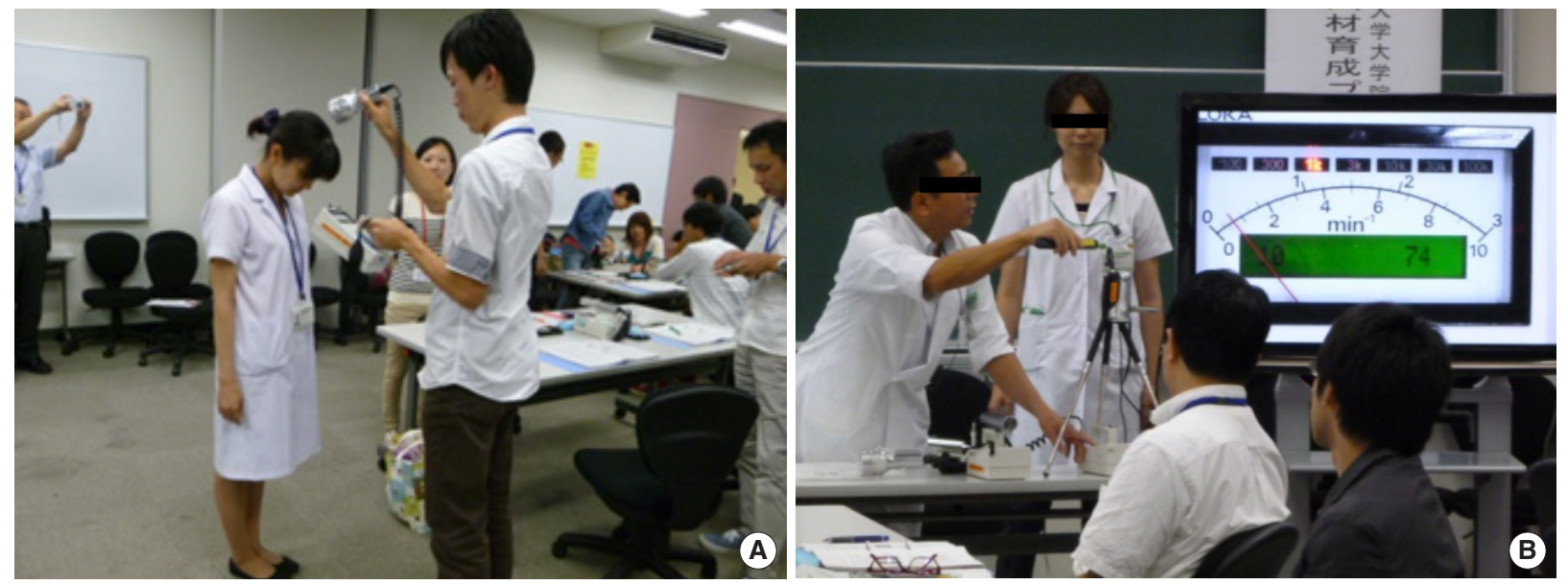

Fig. 1. Hands-on seminar: Radiation detection. (A) All participants learn how to handle radiation detectors and detect contamination. (B) They were also taught the importance of adequately setting of the time constant and speed of survey.

\footnotetext{
4) Advanced Human Resources Development Project for Radiation Emergency Medicine. Outline of the Project. Education for Working Professionals. (internet homepage. Hirosaki University Graduate School of Health Sciences, Special Expenditures Project of Ministry of Education, Culture, Sports, Science and Technology. Advanced Human Resources Development Project for Radiation Emergency Medicine) (cited September 06, 2016) Available from: http://www.hs.hirosaki-u. ac.jp/ hibaku/en/04_program.html.
} 
other during an emergency.

\section{Educational program}

The program of our annual training course is shown in Table 1. The e-learning course comprises five lectures and four movies. The two-day course comprises three lectures, three hands-on seminars, three drills, and one drill review.

The e-learning lectures cover radiation basics and radiation effects, and provide an outline of REM. This two-day training course offers lectures aimed at gaining an in-depth knowledge of each specialty from the perspective of REM, as well as drills designed to help nurses and technologists manage irradiated and/or contaminated patients. The lectures also cover mental health problems associated with nuclear

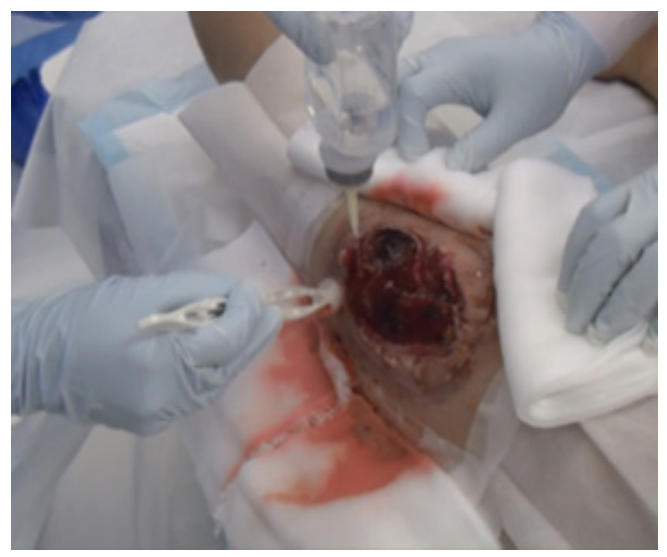

Fig. 2. Hands-on seminar: Decontamination. All participants were divided into small groups of two nurses and two radiological technologists and taught how to detect contamination and decontaminate wound contamination using moulage. power plant (NPP) accidents and a history of serious radiological incidents. The lecture entitled 'Current Situation in Fukushima Prefecture' was offered by a doctor from Fukushima Medical School. Desktop drills include team organization for REM and drill reviews. The hands-on seminar and drills include radiation detection (including how to handle radiation detectors) (Figure 1), decontamination (Figure 2), and medical team drills for NPP accidents (Figure 3). Through these drills, all participants acquire expertise on how to decontaminate and how to put on and take off protective clothing. All participants learn to assess and decontaminate contaminated wounds.

\section{Scenario of medical treatment drill}

In the reactor building of a NPP, a crack occurs in the pipe of the primary coolant. Control rods are inserted and, after cooling, some workers are working to repair the pipe in the reactor building. Two of these workers, who are suffering from the high temperature and humidity, finally collapse at the steps and consequently have wound contamination in their lower legs. Their consciousness levels are slight cloudy, but they have no nausea, vomiting, or fever.

The two contaminated patients are transferred and the participants, who have been divided into groups of 2-4, are put in charge of them. The roles assigned in each group were as follows: two nurses were in charge of patients' treatment and decontamination in the hot area (controlled area); two radiological technologists were in charge of detecting surface contamination in the hot area (controlled area); two-three nurses were in charge of medical records and medical sup-
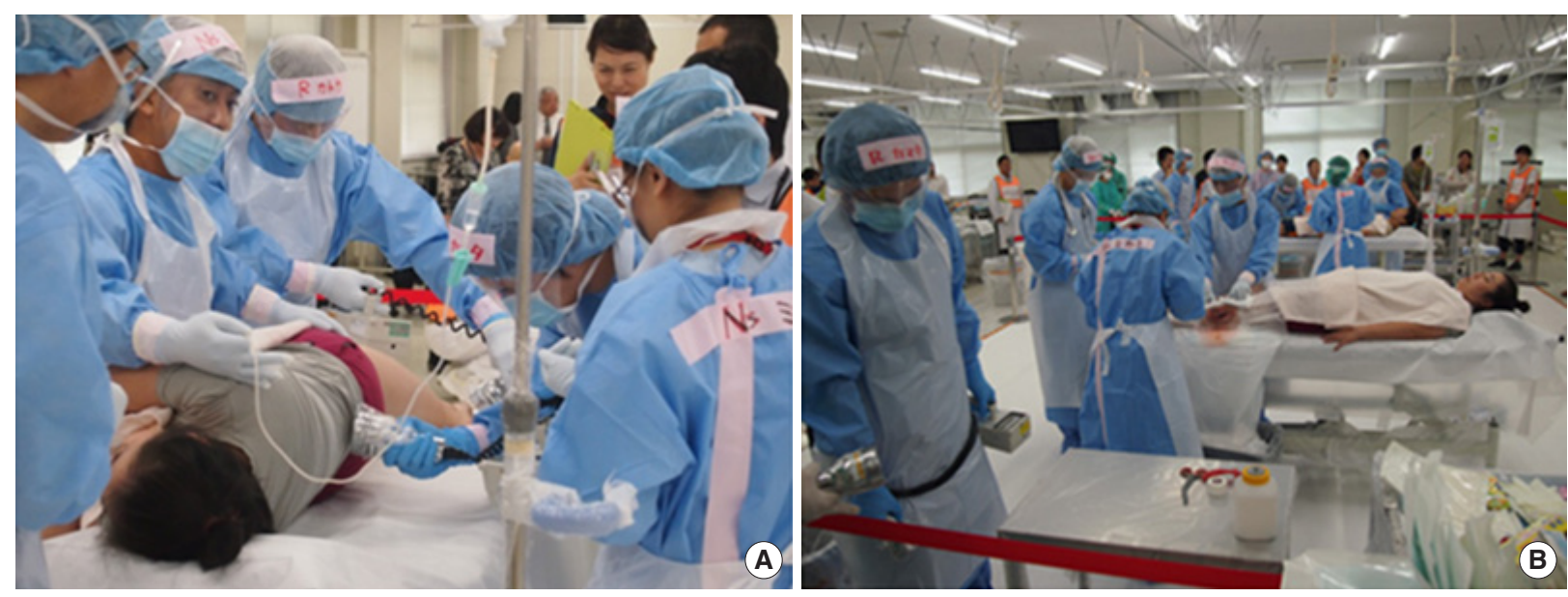

Fig. 3. Medical team drills for NPP accidents. All participants are included in this drill and learn medical treatment for NPP accidents. (A) They detect contamination and $(\mathrm{B})$ decontaminate wound contamination. 
plies outside of the hot area; two radiological technologists were in charge of air dose monitoring and biological sample checks. Some medical doctors in our faculty helped the participants with regard to medical decision making; however, in general, all procedures were performed by participants. Our staff also answered participants' questions and gave them advice during the drill. Participants were required to treat patients according to the concept of REM described in the REAC/TS Patient Treatment Algorithm (Figure 4). ${ }^{5)}$ This concept was taught in e-learning lectures. If a life-threatening problem occurs, medical treatment to stabilize the patient condition should first be performed. If the patient is stable, a surface contamination survey and decontamination are conducted. A blood sample and swab from the nasal and oral cavities should be obtained for further examination, such as radiation dose evaluation and identification of radionuclide. Participants are also required to check symptoms and their onset time so that they can evaluate the severity of irradiated patients according to the classification of the acute radiation syndrome (Table 2 ). Video-recordings were taken during this drill and shown during lunch break immediately after the drill. This helped participants to check their actions before the drill review.

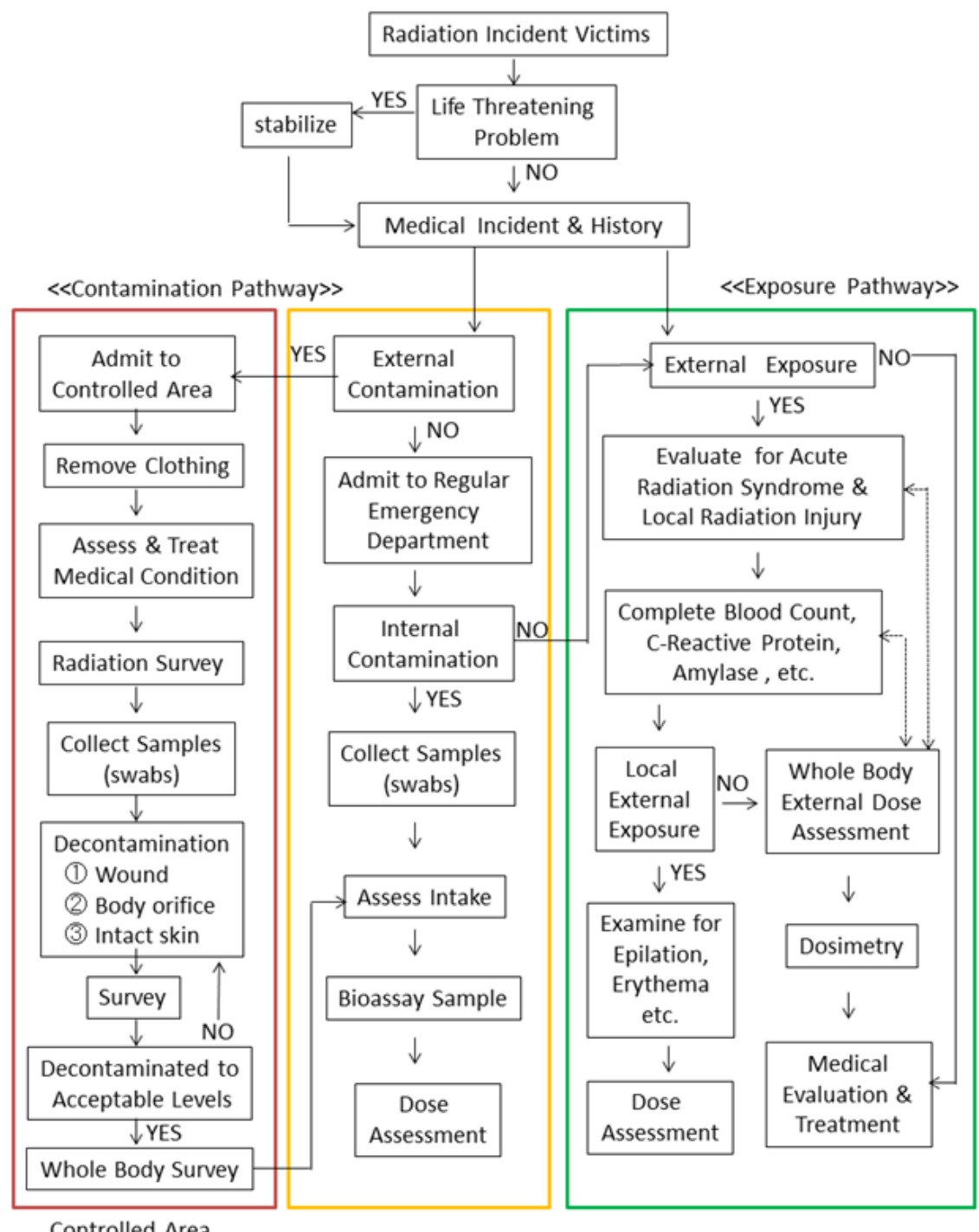

Fig. 4. Patient treatment algorithm in REM Modified from The Medical Aspects of Radiation Incidents in REAC/TS ${ }^{5}$.

5) REAC/TS Patient Treatment Algorithm. (cited September 6, 2016) Available from: https://orise.orau.gov/files/reacts/medical-aspects-of-radiation-incidents.pdf. 
Table 2. Classification of Acute Radiation Syndrome

\begin{tabular}{|c|c|c|c|c|c|c|}
\hline \multicolumn{2}{|c|}{ Symptoms and therapeutic strategy } & \multirow{3}{*}{$\begin{array}{c}\text { Mild 1-2Gy } \\
2 \mathrm{Hr}< \\
10-50 \%\end{array}$} & \multirow{3}{*}{$\begin{array}{c}\text { Moderate 2-4Gy } \\
1-2 \mathrm{Hr}< \\
70-90 \%\end{array}$} & \multirow{3}{*}{$\begin{array}{c}\text { Severe 4-6Gy } \\
1 \mathrm{Hr}> \\
100 \%\end{array}$} & \multirow{3}{*}{$\begin{array}{c}\text { Very severe 6-8Gy } \\
30 \mathrm{Min}> \\
100 \%\end{array}$} & \multirow{3}{*}{$\begin{array}{c}\text { Lethal > } 8 \text { Gy } \\
\text { 10Min > } \\
100 \%\end{array}$} \\
\hline Nausea & Onset time & & & & & \\
\hline & Frequency & & & & & \\
\hline \multirow[t]{2}{*}{ Diarrhea } & Degree & - & - & Mild & Severe & Severe \\
\hline & Frequency & - & - & $<10 \%$ & $>10 \%$ & $100 \%$ \\
\hline \multirow[t]{2}{*}{ Headache } & Degree & Slight & Mild & Moderate & Severe & Severe \\
\hline & Frequency & - & - & $50 \%$ & $80 \%$ & $80-90 \%$ \\
\hline \multirow[t]{2}{*}{ Consciousness } & Degree & Normal & Normal & Normal & May disturbed & Unconscious \\
\hline & Frequency & - & - & - & Few & $100 \%$ \\
\hline \multirow{2}{*}{ Body temperature } & Degree & Normal & Slight fever & Mild fever & High fever & High fever \\
\hline & Frequency & & $10-80 \%$ & $80-100 \%$ & $100 \%$ & $100 \%$ \\
\hline \multicolumn{2}{|l|}{ Therapeutic strategy } & Follow-up & $\begin{array}{l}\text { Admission in } \\
\text { general hosp. }\end{array}$ & $\begin{array}{l}\text { Admission in } \\
\text { special hosp. }\end{array}$ & $\begin{array}{l}\text { Admission in } \\
\text { special hosp. }\end{array}$ & $\begin{array}{l}\text { Advanced medical } \\
\text { treatment }\end{array}$ \\
\hline
\end{tabular}

Table 3. Participant Numbers

\begin{tabular}{lrrrrr}
\hline & 2010 & 2011 & 2012 & 2013 & 2014 \\
\hline Nursing Course & $15(0)$ & $15(0)$ & $12(1)^{\star}$ & $7(0)$ & $15(8)^{\star}$ \\
$\begin{array}{l}\text { Radiological Technologists } \\
\quad \text { Course }\end{array}$ & $3(0)$ & $6(0)$ & $9(4)^{\star}$ & $11(5)^{\star}$ & $16(3)^{\star}$ \\
Total & $18(0)$ & $21(0)$ & $21(5)^{\star}$ & $18(5)^{\star}$ & $31(11)^{\star}$ \\
\hline
\end{tabular}

* (): Participants from other prefectures.

\section{Results and Discussion}

\section{Participant numbers}

The numbers of participants are shown in Table 3. Participants on the Radiological Technologists Course are gradually increasing. Many were from Aomori Prefecture; however, recently, approximately $20-30 \%$ of participants have come from other prefectures.

\section{Participant Achievement and questionnaire survey for participants}

Participants accomplished these drills and exercised, even if it is not perfect. Participants noticed their knowledge and skills related REM through drills. After the training course, participants are supposed to review and make a training plan and prepare for REM in their institutes. The questionnaire survey for participants indicated that participants were satisfied with this training and wanted to attend again (Figure 5).

E-learning was considered effective as it allows repeated learning and study according to participants' schedules. However, some had problems with internet access. These problems will be solved, and information on how to avoid problems should be provided.
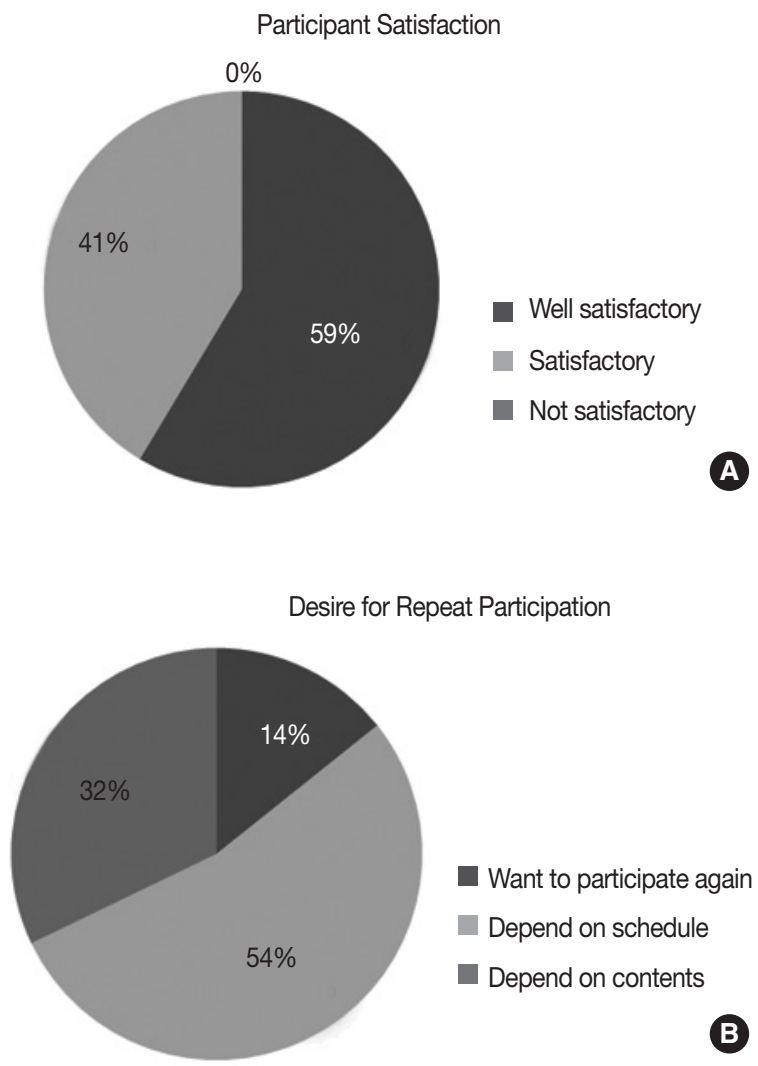

Fig. 5. Questionnaire survey for participants. The questionnaire survey for participants demonstrated that $(A)$ they were satisfied with the training and $(B)$ wanted to attend again.

\section{Features of our training}

Repeated training exercises are thought to be necessary for medical staff to maintain expertise and familiarity with techniques, because nuclear power plant accidents are very rare. Not only institutional training but also multi-institutional trainings should be necessary. Our training participants came from many different institutions throughout Japan. 
The role or missions of each participant and their institutes may differ, and they may notice the different ways or views of other participants in our training. This may help them to broaden their scope.

A great deal of time and effort are required in preparation for this training. Many committee meetings and repeated drill simulations are necessary. However, it is necessary to keep our expertise and we also learned a great deal from our participants through this training course.

Our annual training does not cover nuclear terrorism. However, the basic concepts are the same according to radiation protection and decontamination [1,2]. We believe that this training course will provide a valuable opportunity for medical professionals to gain knowledge and expertise in REM. In future, we are planning to hold another advanced REM training course for repeated participants

\section{Conclusion}

We provide an outline of this education program for working professionals. We believe that this training course will provide a valuable opportunity for medical professionals to gain knowledge and expertise in REM.

\section{References}

1. Saito Y, Kitajima Y, Hosokawa Y, Kudo K, Osanai M,Tsushima M. Report on a civil protection training exercise in Aomori prefecture with a dirty Bomb scenario. Radiat. Emergency Med. 2014; 3(2):31-38.

2. Saga R, Hosokawa Y, Saito Y, Itaki C, Nakamura T. Report on radiation emergency medicine education and training course. Radiat. Environ. Med. 2016;5(2):50-55. 